\title{
Diagnosing Carotid Near-Occlusion with Phase-Contrast MRI
}

\author{
(D)E. Johansson, (D) Zarrinkoob, (D) A. Wåhlin, (D) A. Eklund, and (D). Malm
}

\section{ABSTRACT}

SUMMARY: Carotid near-occlusion is a frequently overlooked diagnosis when CTA examinations are assessed in routine practice. To evaluate the potential value of phase-contrast MR imaging in identifying near-occlusion, we examined 9 carotid near-occlusions and 20 cases of conventional $\geq 50 \%$ carotid stenosis (mean stenosis degree, 65\%) with phase-contrast MR imaging. Mean ICA flow was lower in near-occlusions $(52 \mathrm{~mL} / \mathrm{min})$ than in conventional $\geq 50 \%$ stenosis $(198 \mathrm{~mL} / \mathrm{min}, P<.001)$. ICA flow of $\leq 110 \mathrm{~mL} / \mathrm{min} \mathrm{was}$ $100 \%$ sensitive and specific for near-occlusion. Phase-contrast MR imaging is a promising tool for diagnosing carotid near-occlusion.

ABBREVIATION: $P C=$ phase-contrast

C arotid near-occlusion is a severe carotid stenosis associated with a reduction (collapse) of the extracranial ICA distal to the stenosis. ${ }^{1-4}$ The collapse can be severe (near-occlusion with full collapse; Fig 1A) or subtle (near-occlusion without full collapse; Fig $1 B) .^{1-4}$ Separating symptomatic near-occlusions and conventional stenoses is crucial because their recommended treatments often differ. ${ }^{5,6}$

Near-occlusion is diagnosed by assessing several features on angiography (such as conventional angiography or CTA). Although the feature assessments are reliable among collaborating experts, ${ }^{4}$ implementation in routine practice can be challenging because expertise and feature synthesis likely vary among observers. ${ }^{7}$ Near-occlusion is presumed to be a marker of reduced flow. ${ }^{2}$ Carotid sonography has poor diagnostic performance because there is a nonlinear relationship between the degree of

Received September 16, 2020; accepted after revision December 7.

From the Department of Clinical Science (E.J., L.Z., J.M.), Neurosciences; Wallenberg Center for Molecular Medicine (E.J.); Departments of Perioperative Sciences (L.Z.) and Radiation Sciences (A.W., A.E.); and Umeå Center for Functional Brain Imaging (A.W., A.E.), Umeå University, Umeå, Sweden.

The study was funded by the Swedish Research Council, grant Nos. 2015-05616: 2017-04949; the Knut and Alice Wallenberg Foundation; Region Västerbotten, the Swedish Heart and Lung Foundation, grant No. 20140592; the research fund for neurologic research at the University Hospital of Northern Sweden; the Swedish Stroke Fund; the Northern Swedish Stroke Fund; and the Swedish Medical Association.

Paper preciously presented at: Annual Meeting of the European Society of Neuroradiology, October 5-9, 2020; Virtual.

Please address correspondence to Elias Johansson, MD, Department of Clinical Science, Neurosciences, Umeå University, 901 87, Umeå, Sweden; e-mail: elias.johansson@umu.se

- Indicates open access to non-subscribers at www.ajnr.org

Indicates article with online supplemental data.

http://dx.doi.org/10.3174/ajnr.A7076 stenosis and flow velocity in stenosis. ${ }^{8,9}$ Phase-contrast (PC) MR imaging to assess ICA flow might be useful because it is based on flow, but no PC MR imaging study has assessed ICA flow in near-occlusion.

The aim of this study was to compare ICA flow in carotid near-occlusion and conventional $\geq 50 \%$ stenosis.

\section{MATERIALS AND METHODS}

Patients with symptomatic or asymptomatic $\geq 50 \%$ carotid stenosis at the University Hospital of Northern Sweden were included. Clinical exclusion criteria were atrial fibrillation, any previous central nervous system diseases, $\mathrm{mRS} \geq 3$, Mini-Mental State Examination score of $<23$, an inability to walk unassisted, severe aphasia, or contraindications for MR imaging. Imaging exclusions were any ICA occlusion, intracranial carotid stenosis, uncertainty about whether the index stenosis was conventional or near-occlusion $(n=3)$, and delay between CTA and MR imaging of $>35$ days. The study was approved by the regional ethics board in Umeå. All patients provided informed consent.

\section{CTA}

See the Online Supplemental Data for protocol details. All CTAs were evaluated by 1 observer, and a subset $(n=20)$ was also evaluated by a second observer. Both observers had near-occlusion expertise and were blinded to each other and to flow data. Disagreements were resolved by consensus discussion. Nearocclusion was diagnosed when a severe stenosis was associated with reduction in the distal ICA diameter, as presented elsewhere. ${ }^{4,10,11}$ The definition of full collapse and acknowledging circle of Willis variants are presented elsewhere. ${ }^{4,10}$ In cases without near-occlusion, the degree of stenosis was calculated with the 


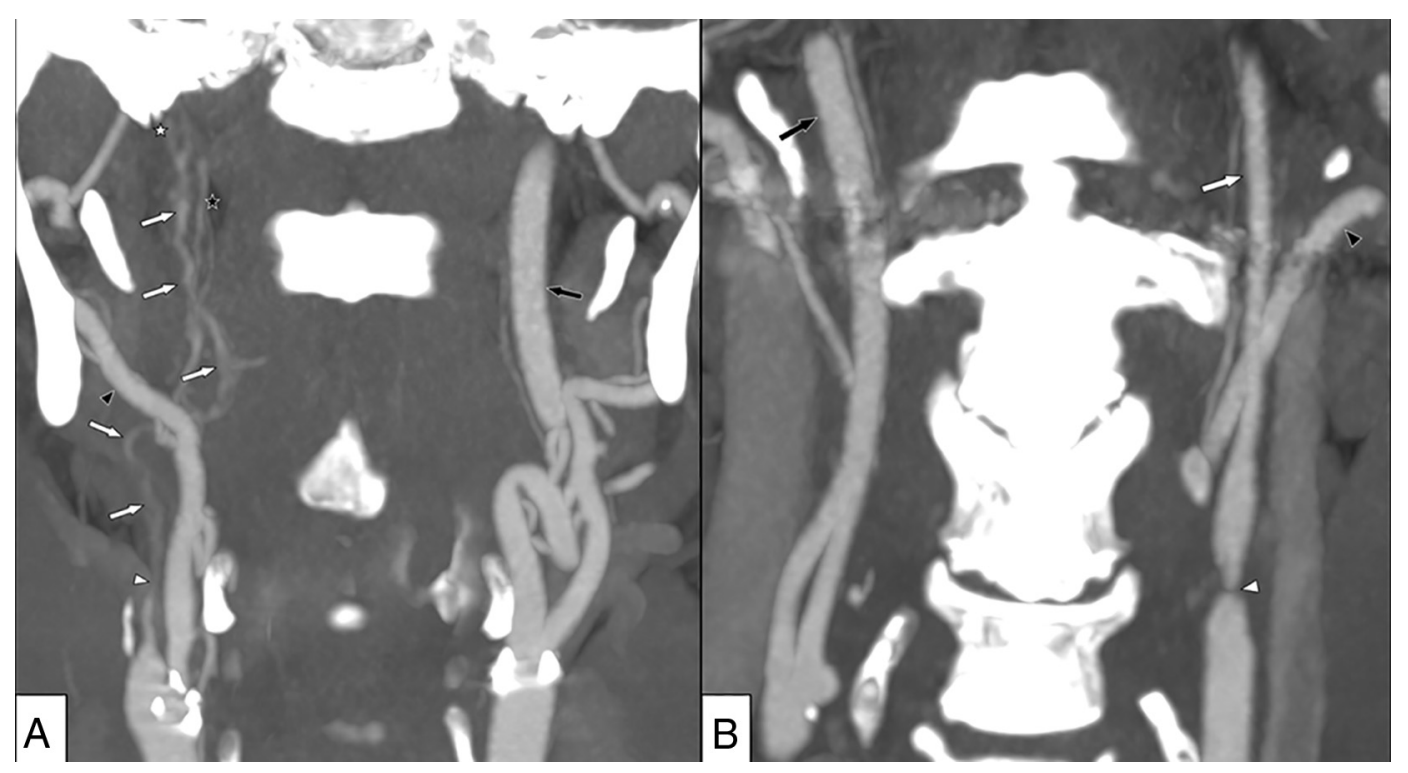

FIG 1. A, Right-sided near-occlusion with full collapse. Coronary view of CTA. Beyond severe stenosis (white arrowhead), distal ICA is difficult to visualize and has a threadlike appearance (white arrows). The distal ICA is clearly smaller than both the contralateral ICA (black arrow) and ipsilateral external carotid artery (black arrowhead) but is similar to the ascending pharyngeal artery (black star). A thrombus just proximal to the skull base is suspected (white star). ICA flow was $6 \mathrm{~mL} / \mathrm{min}$ on the right side and $267 \mathrm{~mL} / \mathrm{min}$ on the left side. $B$, Left-sided near-occlusion without full collapse. Coronary view of CTA. After severe stenosis (white arrowhead), the distal ICA is small but normal-appearing (white arrow), smaller than the contralateral ICA (black arrow), and similar to the ipsilateral external carotid artery (black arrowhead). ICA flow was $37 \mathrm{~mL} / \mathrm{min}$ on the left side and $259 \mathrm{~mL} / \mathrm{min}$ on the right side.

NASCET approach, comparing stenosis diameter with distal ICA well beyond the stenosis.

\section{Phase-Contrast MR Imaging}

A 3T MR imaging scanner (Discovery MR 750; GE Healthcare) with a 32-channel head coil was used. 4D PC MR images were gathered and analyzed with in-house software similar to a recent study (Online Supplemental Data). ${ }^{12,13}$ Two observers blinded to CTA findings and each other extracted flow data from both extracranial ICAs just below the skull base in all cases. The mean value between the 2 observers was used for main calculations. When the observers differed $>20 \%$, consensus measurements were performed. Each observer's measurements were also analyzed.

\section{Analyses}

Patients were categorized by CTA into conventional $\geq 50 \%$ stenosis and near-occlusion. Flow rates in the ICA were compared between stenosis groups, degrees of stenosis, and CTA measurements. Relative flow and area were calculated as ipsilateral/(ipsilateral + contralateral). We used mean, SD, a 2 -sided $\chi^{2}$ test, a $t$ test, linear regression, a receiver operating characteristic curve, $\kappa$ analysis, and intraclass correlation. The $95 \%$ confidence intervals for correlation $(r)$ were calculated with $z$ scores. $P<.05$ was prespecified as statistically significant. We used SPSS 24.0 (IBM) for all calculations.

\section{RESULTS}

In total, we included 29 patients: Twenty (69\%) had conventional $\geq 50 \%$ stenosis, and 9 (31\%) had near-occlusion. Baseline characteristics were similar $(P \geq .13)$ in both groups: The mean age was 73 years, $24 \%$ were women, the mean delay between examinations was 6 days, and $86 \%$ had symptomatic stenosis. Among the conventional stenoses, the mean stenosis degree was $65 \%, 8$ were $\geq 70 \%$, and the remaining degrees were $50 \%-69 \%$. Two cases of conventional stenoses had asymmetric distal ICAs, best explained by circle of Willis asymmetry. Eight cases had bilateral $\geq 50 \%$ stenosis, but there were no cases with bilateral near-occlusion.

The mean ICA flow was lower in near-occlusions $(52 \mathrm{~mL} /$ min) than in conventional $\geq 50 \%$ stenosis $(198 \mathrm{~mL} / \mathrm{min}, P<$ $.001)$. The mean relative ICA flow was lower in near-occlusions (16\%) than in conventional $\geq 50 \%$ stenosis (48\%, $P<.001$ ). Neither parameter had overlap between the groups (Online Supplemental Data). Thus, several thresholds, such as ICA flow $\leq 110 \mathrm{~mL} / \mathrm{min}$ and relative flow $<35 \%$, were $100 \%$ sensitive and specific for near-occlusion. One case had near-occlusion with full collapse, with a $6 \mathrm{~mL} / \mathrm{min}$ ICA flow.

Among the 20 patients with conventional $\geq 50 \%$ stenosis, there was no correlation between percentage degree of stenosis and ICA flow ( $r=0.06$; 95\% CI, $-0.44-0.55 ; P=.81$ ). There was no difference in the mean ICA flow between the 12 patients with $50 \%-69 \%$ stenoses $(199 \mathrm{~mL} / \mathrm{min})$ and the 8 patients with $\geq 70 \%$ stenoses $(197 \mathrm{~mL} / \mathrm{min}, P=.90)$. However, when analyzing all 29 patients, there was a correlation between stenosis diameter and ICA flow ( $r=0.68$; 95\% CI, 0.39-0.96; $P<.001$ ). A similar correlation was noted for the ipsilateral distal ICA area and ipsilateral ICA flow ( $r=0.69$; 95\% CI, 0.42-0.96; $P<.001$ ). Relative distal ICA area and relative ICA flow were even more strongly correlated $(r=0.90$; 95\% CI, 0.74-1.00; $P<.001$ ). Please see the Online Supplemental Data for figures of these regression analyses.

Overall agreement between the blinded CTA observers was 96\% (1 disagreement over whether there was occlusion), and interrater reliability was $\kappa=0.78$. The 2 PC MR imaging observers had a consensus discussion over 17 (29\%) neck sides but 100\% agreement 
as to whether ICA flow and relative ICA flow were above or below the proposed diagnostic thresholds $(110 \mathrm{~mL} / \mathrm{min}$ and $35 \%$, respectively), and the intraclass correlation coefficient was 0.96 (Online Supplemental Data).

\section{DISCUSSION}

The main findings of this study were that carotid near-occlusions had low ipsilateral ICA flow, whereas conventional $\geq 50 \%$ carotid stenoses had no correlation between the degree of stenosis and ICA flow $(r=0.06)$. Flow was correlated with stenosis diameter and distal ICA area, both hallmarks of near-occlusion.

No single feature in CTA is both $>90 \%$ sensitive and specific for near-occlusion; thus, several features are used for angiographic diagnosis. ${ }^{3,11}$ Flow measurement seems more accurate, but a larger sample will be needed for a relevant assessment. According to guidelines, patients with symptomatic conventional stenosis should be treated with revascularization. ${ }^{5,6}$ However, patients with symptomatic near-occlusions should not be treated with revascularization $^{6}$ or only treated after careful consideration in cases with recurrent events despite optimal medical therapy. ${ }^{5}$ To separate conventional stenosis and near-occlusion, there might be a role for PC MR imaging in routine carotid stenosis diagnostics after the stenosis has been identified by another technique. See the Online Supplemental Data for details on PC MR imaging literature. The association between near-occlusion and flow might also improve understanding of prognostics ${ }^{4,14}$ and sonography findings, ${ }^{8,15}$ in near-occlusion. Other PC MR imaging techniques (2D and 3D) are likely to produce similar results, but they should be evaluated.

The concepts of near-occlusion and conventional stenoses are limited to NASCET grading. If near-occlusions are not recognized, percentage grading with NASCET leads to stenosis underestimation. Stenosis diameter was correlated with flow, as expected from the Spencer curve model. ${ }^{9}$ However, the Spencer model assumes a stable denominator, ${ }^{9}$ which is not the case in NASCET grading. When stenoses were graded with the NASCET method, only near-occlusions affected flow, not the conventional stenoses regardless of percentage grade. The ICA flow of conventional stenoses was rather similar in healthy elderly. ${ }^{13}$

This work has several limitations: The sample size was small. The near-occlusion prevalence (31\%) might be perceived to be high but was similar (27\%) to that in a recent larger study using the same diagnostic approach. ${ }^{4}$ We excluded cases with contralateral occlusion and those that were unclear as to whether the degree of stenosis was conventional $\geq 50 \%$ stenosis or near-occlusion. Thus, although the results are promising, large studies and studies dedicated to the cases now excluded and bilateral nearocclusion are warranted.

\section{CONCLUSIONS}

When we used NASCET-grading, only near-occlusions had a reduced ICA flow, while ICA flow was not affected by conventional stenoses. Because near-occlusion has a different clinical management than conventional stenosis and near-occlusion is difficult to diagnose with CTA, there is support for including ICA PC MR imaging in the imaging protocol for patients with ICA stenosis.

\section{ACKNOWLEDGMENTS}

We want to thank Dr Allan J. Fox for help with CTA interpretations and assistance with manuscript preparations, including details about NASCET grading methodology.

Disclosures: Elias Johansson—RELATED: Grant: several grants to my institution, listed in funding section*; UNRELATED: Royalties: Studentlitteratur, Comments: 2 book chapters in 2014 in a medical textbook on stroke; generates annual royalties. Anders Eklund-RELATED: Grant: Swedish Research Council.* *Money paid to the institution.

\section{REFERENCES}

1. Johansson E, Fox AJ. Carotid near-occlusion: a comprehensive review, Part 1: definition, terminology, and diagnosis. AJNR Am J Neuroradiol 2016;37:2-10 CrossRef Medline

2. Johansson E, Fox AJ. Carotid near-occlusion: a comprehensive review, Part 2: prognosis and treatment, pathophysiology, confusions, and areas for improvement. AJNR Am J Neuroradiol 2016;37: 200-04 CrossRef Medline

3. Fox AJ, Eliasziw M, Rothwell PM, et al. Identification, prognosis, and management of patients with carotid artery near occlusion. AJNR Am J Neuroradiol 2005;26:2086-94 Medline

4. Gu T, Aviv RI, Fox AJ, et al. Symptomatic carotid near-occlusion causes a high risk of recurrent ipsilateral ischemic stroke. J Neurol 2020;267:522-30 CrossRef Medline

5. Naylor AR, Ricco JB, de Borst GJ, et al. Management of Atherosclerotic Carotid and Vertebral Artery Disease: 2017 Clinical Practice Guidelines of the European Society for Vascular Surgery (ESVS). Eur J Vasc Endovasc Surg 2018;55:3-81 CrossRef Medline

6. Brott TG, Halperin JL, Abbara S, et al. Society for Vascular Surgery. ASA/ACCF/AHA/AANN/AANS/ACR/ASNR/CNS/SAIP/SCAI/SIR/ SNIS/SVM/SVS Guideline on the Management of Patients with Extracranial Carotid and Vertebral Artery Disease. Circulation 2011;124:e54-130 CrossRef Medline

7. Johansson E, Gu T, Aviv RI, et al. Carotid near-occlusion is often overlooked when CT-angiography is assessed in routine practice. Eur Radiol 2020;30:2543-51 CrossRef Medline

8. Khangure SR, Benhabib H, Machnowska M, et al. Carotid nearocclusion frequently has high peak systolic velocity on Doppler ultrasound. Neuroradiology 2018;60:17-25 CrossRef Medline

9. Spencer MP, Reid JM. Quantitation of carotid stenosis with continuous-wave (C-W) Doppler ultrasound. Stroke 1979;10:32630 CrossRef Medline

10. Johansson E, Aviv RI, Fox AJ. Atherosclerotic ICA stenosis coincidental with ICA asymmetry associated with circle of Willis variations can mimic near-occlusion. Neuroradiology 2020;62:101-04 CrossRef Medline

11. Bartlett ES, Walters TD, Symons SP, et al. Diagnosing carotid stenosis near-occlusion by using CT angiography. AJNR Am J Neuroradiol 2006;27:632-37 Medline

12. Zarrinkoob L, Wåhlin A, Ambarki K, et al. Blood flow lateralization and collateral compensatory mechanisms in patients with carotid artery stenosis. Stroke 2019;50:1081-88 CrossRef Medline

13. Zarrinkoob L, Ambarki K, Wåhlin A, et al. Blood flow distribution in cerebral arteries. J Cereb Blood Flow Metab 2015;35:648-54 CrossRef Medline

14. Johansson E, Öhman K, Wester P. Symptomatic carotid near-occlusion with full collapse might cause a very high risk of stroke. $J$ Intern Med 2015;277:615-23 CrossRef Medline

15. Johansson E, Benhabib H, Herod W, et al. Carotid near-occlusion can be identified with ultrasound by low flow velocity distal to the stenosis. Acta Radiol 2019;60:396-404 CrossRef Medline 